University of Nebraska - Lincoln

DigitalCommons@University of Nebraska - Lincoln

Faculty Publications in Computer \& Electronics Electrical \& Computer Engineering, Department Engineering (to 2015)

2011

\title{
Priority Preemption for Real-time Application QoS Guarantees in Cooperative Vehicular Networks
}

\author{
Ting Zhou \\ University of Nebraska-Lincoln, tzhou@unInotes.unl.edu \\ Hamid Sharif \\ University of Nebraska-Lincoln, hsharif@unl.edu \\ Michael Hempel \\ University of Nebraska-Lincoln, mhempel2@unl.edu \\ Puttipong Mahasukhon \\ University of Nebraska-Lincoln, pmahasukhon@huskers.unl.edu \\ Tao Ma \\ University of Nebraska Lincoln, tma@unInotes.unl.edu \\ See next page for additional authors
}

Follow this and additional works at: https://digitalcommons.unl.edu/computerelectronicfacpub

Part of the Computer Engineering Commons

Zhou, Ting; Sharif, Hamid; Hempel, Michael; Mahasukhon, Puttipong; Ma, Tao; and Shrestha, Pradhumna, "Priority Preemption for Real-time Application QoS Guarantees in Cooperative Vehicular Networks" (2011). Faculty Publications in Computer \& Electronics Engineering (to 2015). 106.

https://digitalcommons.unl.edu/computerelectronicfacpub/106

This Article is brought to you for free and open access by the Electrical \& Computer Engineering, Department of at DigitalCommons@University of Nebraska - Lincoln. It has been accepted for inclusion in Faculty Publications in Computer \& Electronics Engineering (to 2015) by an authorized administrator of DigitalCommons@University of Nebraska - Lincoln. 


\section{Authors}

Ting Zhou, Hamid Sharif, Michael Hempel, Puttipong Mahasukhon, Tao Ma, and Pradhumna Shrestha 


\title{
Priority Preemption for Real-time Application QoS Guarantees in Cooperative Vehicular Networks
}

\author{
Ting Zhou, Hamid Sharif, Michael Hempel, Puttipong Mahasukhon, Tao Ma, Pradhumna Shrestha \\ Computer and Electronics Engineering Department \\ University of Nebraska-Lincoln \\ Omaha, NE USA \\ \{tzhou, hsharif, mhempel,pmahasukhon, tma, plshrestha\}@ unlnotes.unl.edu
}

\begin{abstract}
Inter-vehicle and roadside-to-vehicle communications can contribute to a safer and more efficient driving experience by providing time-sensitive and location-aware information. However, its performance suffers from vehicle mobility, intermittent user connectivity, and wireless channel unreliability. In this paper, we propose a novel cross-layer optimization approach based on our Adaptive Distributed Cooperative Medium Access Control (ADC-MAC) protocol to guarantee the quality-of-service $(\mathrm{QoS})$ of real-time applications. Markov chain based theoretical analysis show that our proposed priority preemption approach can improve the quality of a real-time application by guaranteeing its bandwidth and reducing its transmission latency.
\end{abstract}

Keywords- Cross Layer, Protocol Optimization, Cooperative Relaying, QoS, Vehicular Networks

\section{INTRODUCTION}

In the foreseeable future, most new vehicles will be equipped with wireless radio device, which will provide time-sensitive and location-aware information to drivers and other vehicles from localized traffic updates to warning signals. Such timely information conveyed over a vehicular network is envisioned to help significantly reduce the number of annual traffic fatalities and injuries [1].

The basic idea of cooperative relaying is that a wireless station with low data rate can achieve higher performance with the help of neighboring stations with high data rate if available. These assisting nodes are referred to as relay nodes or helper stations. A concrete example is shown in Fig. 1: vehicles $B$ and $C$ can communicate with its service access point (AP 0) directly to get the maximum throughput, while vehicle $\mathrm{B}$ can only achieve its maximum throughput with the assistance of vehicle C. Even worse, without the relay service provided by vehicle $B$, vehicle A cannot communicate at all with access points (AP).

Thus far, only few research papers have been

Manuscript submitted at September 30, 2010. This work was supported by a grant from the Federal Railroad Administration (FRA) under Grant 25-1105. The support provided to us by BNSF and Union Pacific Railway.

The authors are with the Telecommunications Engineering Laboratory (TEL), Department of Computer and Electronics Engineering, University of Nebraska-Lincoln, Omaha, NE 68182 USA (e-mail: hsharif@unl.edu).

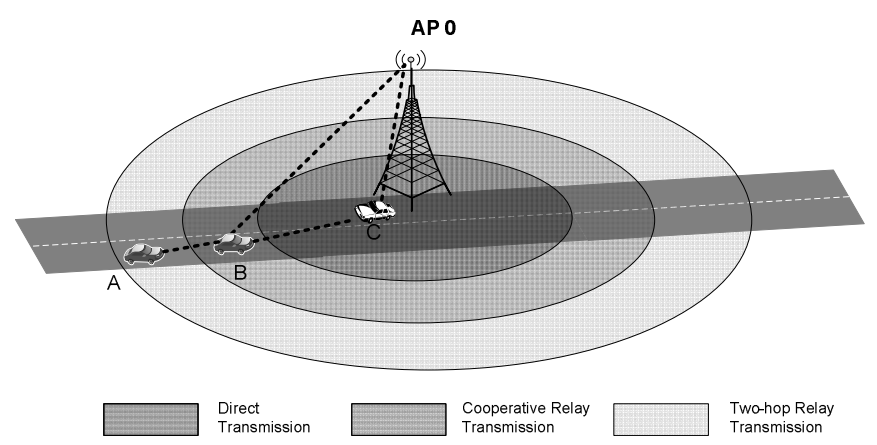

FIGURE 1: Application scenario

published for cooperative MAC protocol design. Relayenabled DCF (rDCF) is firstly proposed in [2] to exploit the multi-rate capability of IEEE 802.11 [3], which can intelligently apply two-hop data transmission to achieve higher throughput by triangular handshakes among a transmitter, a receiver and a predetermined helper. CoopMAC I and II are presented in [4-5] to study the performance impact of cooperation on inter-cell interference. In CoopMAC, each mobile station and AP maintains a table, referred as CoopTable, which includes a set of candidate helpers for data transmission assistance.

To dynamically select relay nodes, another relayenabled MAC protocol is proposed in [6]. In this scheme, after receiving request-to-send (RTS) and clear-to-send (CTS) packets, in according with the channel condition, a helper transmits ready-to-relay (RTR) packets to declare that it has the ability to relay. However, when there are coexisting two or more qualified relay nodes, a collision will unavoidably occur. Another approach is vehicular cooperative media access control (VC-MAC) protocol, which is proposed in [7-8]. It assumes that all vehicles within an AP's coverage range can be synchronized by receiving data packets from that AP, so it is only suitable for downlink traffic scenarios. Because the whole network needs to synchronize once for each data transmission, the overhead of VC-MAC is much heavy and in the worst case, only less than 10 per cent of the channel bandwidth can be used to transmit data.

Therefore the aforementioned studies shown in the above are not optimal for vehicular networks and not 


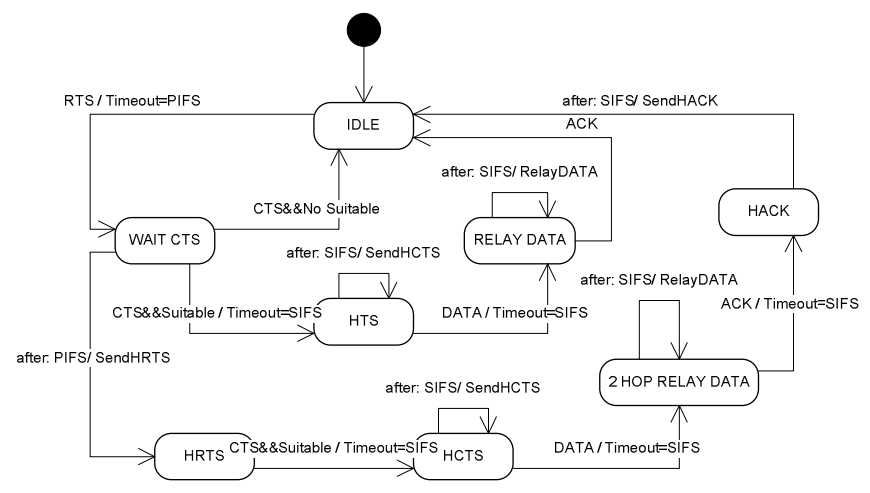

FIGURE 2: The state machine of ADC-MAC protocol

able to sufficiently exploit spatial diversity for mobile wireless communications.

The motivation of our research is to design a new adaptive distributed cooperative relay MAC protocol to improve the achieved system throughput, maximize the service range and guarantee the real-time application QoS in vehicular networks.

In this paper, a novel cross-layer QoS guarantee approach based on our proposed ADC-MAC protocol [9] is presented. By exchanging both information of the channel condition in the physical layer and the QoS requirement knowledge in the application layer, higher priority traffic can interrupt the current data transmission of lower priority traffic to preempt the bandwidth resource for its communications. So by this way, the service quality of real-time applications in vehicular networks can be guaranteed. We also validated the performance of our proposed approach by Markov chain based theoretical modeling. The analytical results show that the proposed priority preemption approach can improve the quality of a realtime application by guaranteeing its bandwidth and reducing its transmission latency.

The remainder of this paper is structured as follows. The proposed priority preemption approach is presented in Section II while its theoretical performance analysis is presented in Section III. Finally, section IV concludes this paper.

\section{PRIORITY PREEMPTION SCHEME BASED ON ADC-MAC PROTOCOL}

The framework of our proposed fully adaptive distributed cooperative medium access control (ADCMAC) protocol has been presented in [9]. The key idea of this protocol is coordinating cooperative relay activities by RTS-CTS-HTS triangular handshake to choose the most suitable transmission mode among direct transmission (DT), cooperative relay (CR) transmission and two-hop relay (TR) transmission and the most suitable helper for assistance during data transmissions. The key features of ADC-MAC protocol are fully distributed, which does not depend on time synchronization among nodes within the entire network, and self-learning, which predetermined knowledge is not necessary.

ADC-MAC is designed to be backward-compatible with the IEEE 802.11 protocol, so that it can be deployed in coexistence scenarios, which are side-byside with traditional IEEE 802.11 networks and cooperative-relay enhanced vehicular wireless networks. Its internal state machine is shown in Fig. 2.

There are two stages for each data transmission in ADC-MAC protocol: Three party handshake stage and data transmission stage. The first stage is used to select the most suitable transmission mode and the most suitable helper for data transmission. In the first stage, all handshake packets are transmitted with the basic data rate.

The transmitter detects the channel state by the physical carrier sensing. If the received signal power is constantly lower than the given time interval, it will consider that the channel is idle and send an RTS packet to the receiver, which includes an optional helper candidate address field.

After a SIFS interval upon receiving this RTS packet, the receiver will respond with a CTS packet back to the sender. An additional RTS-SNR field is attached in CTS packets to report the signal-to noise ratio (SNR) value of the received RTS packets. A helper candidate address field is optional if the receiver recommends a better helper for assistance.

When a relay node's address matches the helper candidate address field of the received CTS packet, and the SNR values of the received RTS and CTS packets are greater than the given threshold, it will declare that it has the ability to relay data by transmitting an HCTS packet, which includes the RTS-SNR field and the CTS-SNR field to report the SNR value of the received RTS and CTS packets. When the transmitter receives this HCTS packet, if the value of RTS-SNR field and the value of the CTS-SNR field are both greater than the given Relay-SNR threshold, the transmitter will choose this relay node as its helper; otherwise it will send DATA packets to the receiver directly.

When a relay node's address matches the helper candidate address field of the received RTS packet, if during a PIFS interval, the channel is still idle, it will send a helper-request-to-send (HRTS) packet to trigger TR transmission mode. If the receiver gets this HRTS and misses the previous RTS packet, it shall response a CTS packet. The relay node will send a helper-clear-tosend (HCTS) packet to the transmitter as soon as receiving this CTS packet. By this way, the transmitter knows that it can relay data to the receiver through this relay node. When the relay node receives an acknowledge (ACK) packet from the receiver, it will transmit a helper-acknowledge (HACK) to the transmitter. From the HACK sent by the helper, the transmitter will ensure that the current data transmission is successful. This mechanism in effect provides an extended coverage. 

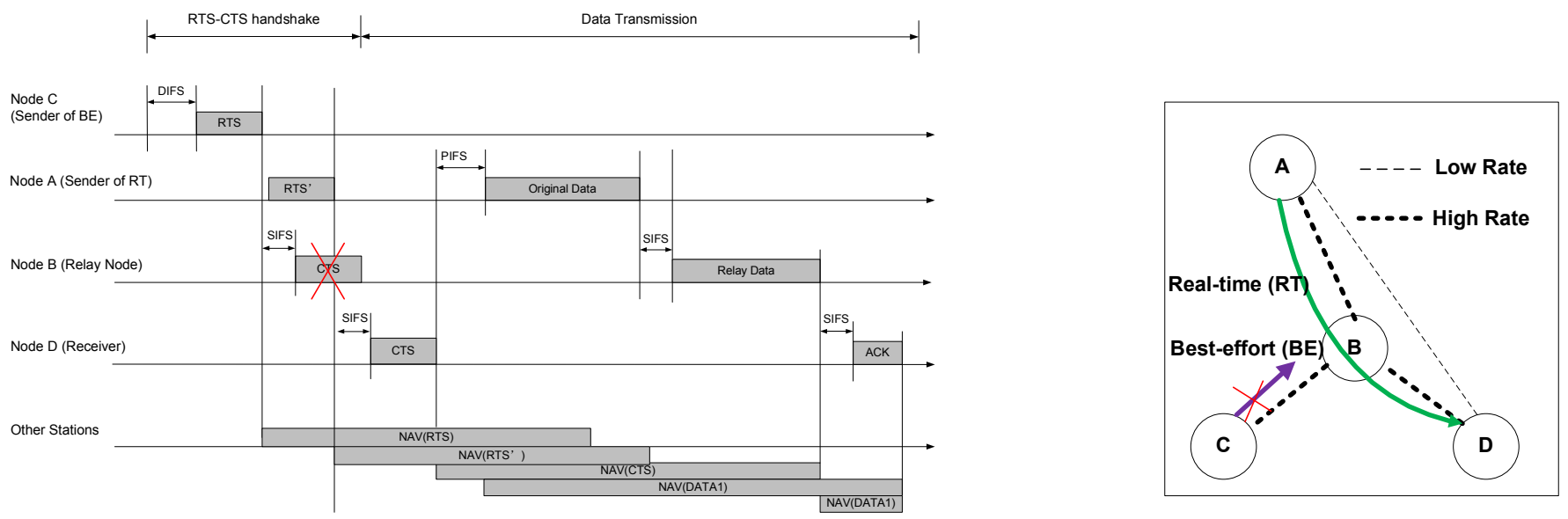

(a)

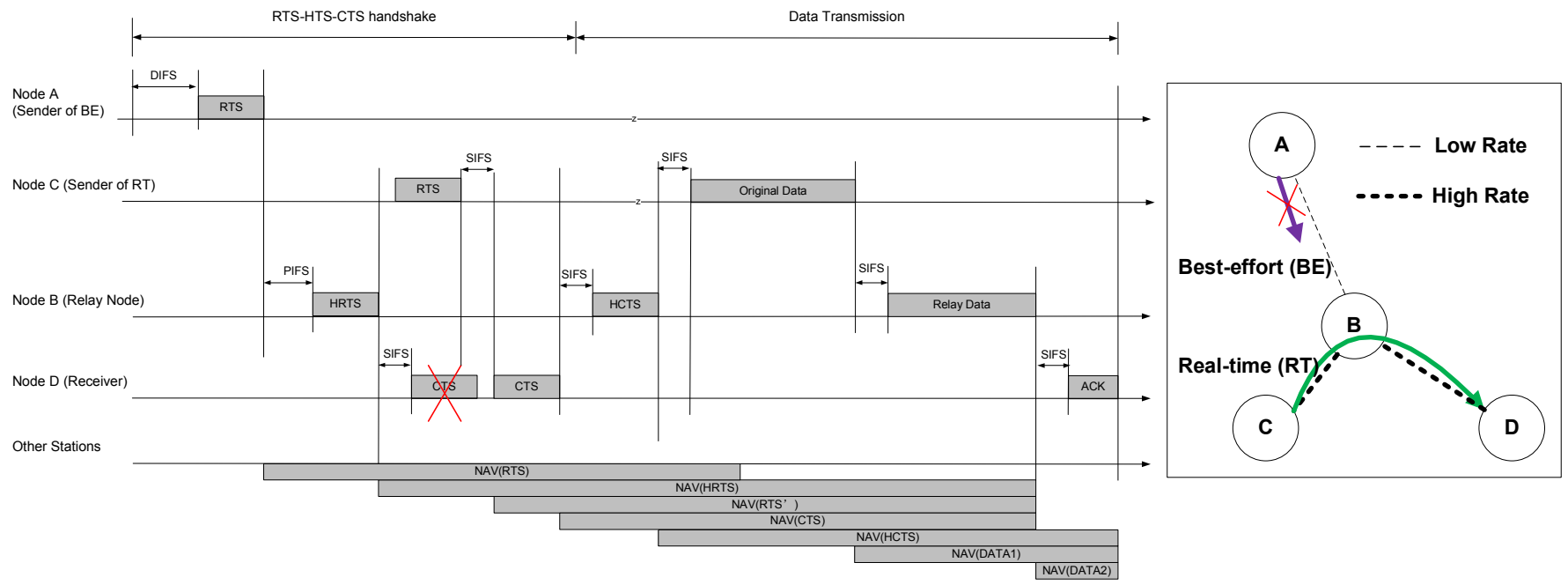

(b)

FIGURE 3: the priority preemption timeline examples in CR and TR mode

Via the above three-party handshake process, a transmitter can exploit diversity gain to select the most suitable transmission mode and the most suitable helper to send DATA frames.

Based on our proposed ADC-MAC protocol, to guarantee the quality of real-time applications, we design a priority preemption scheme to allow a higher priority traffic interrupt the current handshake owned by lower priority traffic by sending a modified RTS packet within this SIFS interval, and then start a new one.

The modified format of RTS packet contains a duration field that reports the transmitting time for the following DATA frame, and a priority field to notify if the data is best-effort or real-time application.

Higher priority traffic with a shorter contention window size and can send a RTS packet within this SIFS interval upon receiving a RTS packet by the transmitter of the lower priority traffic to interrupt the current handshake and restart a new one. Also in TR mode, if the priority of the current traffic is best effort, the current handshake may be canceled when another transmitter sends a RTS packet within this SIFS interval upon receiving the HRTS packet. Fig. 3-(a) shows how a real-time (RT) traffic (between Node A and Node D) interrupts the current transmission of best effort (BE) traffic (between Node C and Node D) and preempts the bandwidth to start its new data transmission in CR mode, which Node A, the sender of the RT traffic can received the broadcasted RTS packet from Node $\mathrm{C}$ directly. In TR mode, Node $\mathrm{C}$ cannot receive the broadcasted RTS packet from Node A directly, however it can interrupt the current $\mathrm{BE}$ traffic transmission between Node A and Node D as soon as it receives a HRTS packet from Node B (Fig. 3-(b)).

By the above priority preemption scheme, our proposed MAC protocol design can utilize the characteristics of vehicular networks and take fully into account the impact of spatial diversity and application QoS requirements. 


\section{THEORETICAL ANALYSIS}

In this section the theoretical throughput and delay performance of the proposed priority preemption approach based on ADC-MAC protocol is evaluated by Markov based modeling, which mathematically evaluates the performance of the Distributed Coordination Function (DCF) in IEEE 802.11 protocol [10-14]. The influences of backoff windows, collision probability, retransmission limitation, and channel bit error rate are taken into consideration.

Without loss of generality and maintaining tractability of the analytical performance model of the IEEE 802.11 protocol and other existing cooperative relaying protocols, we stipulate the following assumptions for our design and theoretical analysis:

- $\quad$ Each node only has one MAC interface, which cannot transmit and receive at the same time;

- The packet transmissions among all nodes share the same medium;

- When receiving a packet, a station can also obtain the physical layer information of that packet, such as received SNR value, noise floor, channel number, by reading corresponding registers, which is similar to the functionalities provided by Atheros baseband process chipsets [15];

- The network will consist of a single source, a destination and some potential helper nodes. This role assignment is time varying;

- The MAC header and the data payload are transmitted with data rate $R_{d}$ (in bits per second) while the physical layer preamble, management and control packets (i.e., Beacon, RTS, CTS, HRTS, HCTS, HACK and ACK packets) are transmitted with basic data rate $R_{C}$ (in bits per second);

- The channel is prone to error and the channel bit error $P_{b}$ is uniformly distributed with error events being independent of each other;

- There is no error correction mechanism in the physical layer;

- $\quad$ Each node transmits packets with probability $\tau$;

- Omni-directional antennas are employed

The normalized throughput $\mathrm{S}$ can be expressed as the ratio

$$
S=\frac{E[p]}{E[s l o t]}
$$

where $E[$.$] is the expectation operator, E[p]$ is the average transmitted payload length in a slot time and $E[$ slot $]$ is the average length of a slot time.

Let $P_{\mathrm{t}}$ be the probability that the channel is busy. This means that there is at least one transmission in the considered slot time. If each of the $n$ stations transmits a packet with probability $\tau$, we can get the following equation:

$$
P_{t}=1-(1-\tau)^{n}
$$

Let Ps be the probability of a non-collision transmission.

$$
P_{s}=\frac{n \tau(1-\tau)^{n-1}}{1-(1-\tau)^{n}}
$$

Let $P_{\mathrm{e}}$ be the probability that the transmission failure is caused by channel bit errors. Since we assume that channel bit errors are uniformly distributed and error events are independent we can express $P_{\mathrm{e}}$ of our ADC-MAC protocol for the cases of direct transmission, cooperative relaying and 2-hop relaying, respectively, as:

$$
\begin{aligned}
& P_{e-D T}=1-\left(1-P_{b}\right)^{L_{D T}} \\
& P_{e-C R}=1-\left(1-P_{b}\right)^{L_{R T S}+L_{C T S}+L_{H C T S}+L_{D A T A}+L_{D A T A}+L_{A C K}} \\
& P_{e-T R}=1-\left(1-P_{b}\right)^{L_{R T S}+L_{H R T S}+L_{C T S}+L_{H C T S}+L_{D A T A}+L_{D A T A}+L_{A C K}+L_{H A C K}}
\end{aligned}
$$

where $L_{R T S}, L_{C T S}, L_{D A T A}, L_{A C K}, L_{H R T S}, L_{H C T S}$ and $L_{H A C K}$ are the length of the RTS, CTS, DATA, ACK, HRTS, HCTS, HACK packets, respectively.

For our proposed protocol, without priority preemption, $S$ can therefore be expressed as:

$$
\mathrm{S}=\frac{P_{t r} P_{s}\left(1-P_{e}\right) E[P]}{\left(1-P_{t r}\right) \sigma+P_{t r} P_{s}\left(1-P_{e}\right) T_{s}+P_{t r} P_{s} \mathrm{H}_{\mathrm{e}}+P_{t r}\left(1-P_{s}\right) T_{c}}
$$

where $T_{P}$ is the average payload transmit time, $T_{S}$ and $T_{c}$ are the average time of a successful transmission and a collision, respectively, and $\sigma$ is the duration of a slot time. $H_{\mathrm{e}}$ is the network overhead due to channel errors, in which packets received with bit errors have been dropped.

$$
\begin{gathered}
\mathrm{H}_{e-D T}=P_{e}^{r t s} \mathrm{~T}_{e}^{r t s}+P_{e}^{c t s} \mathrm{~T}_{e}^{c t s}+P_{e}^{\text {data }} \mathrm{T}_{e}^{\text {data }}+P_{e}^{\text {ack }} \mathrm{T}_{e}^{\text {ack }} \\
\mathrm{H}_{e-C R}=P_{e}^{r t s} \mathrm{~T}_{e}^{r t s}+P_{e}^{c t s} \mathrm{~T}_{e}^{c t s}+P_{e}^{\text {hts }} \mathrm{T}_{e}^{\text {hcts }}+P_{e}^{\text {data }} \mathrm{T}_{e}^{\text {data }}+P_{e}^{\text {ack }} \mathrm{T}_{e}^{\text {ack }} \\
\mathrm{H}_{e-T R}=P_{e}^{r t s} \mathrm{~T}_{e}^{r t s}+P_{e}^{\text {hrts }} \mathrm{T}_{e}^{\text {hrts }}+P_{e}^{\text {cts }} \mathrm{T}_{e}^{\text {cts }}+P_{e}^{\text {hcts }} \mathrm{T}_{e}^{\text {hcts }}+ \\
P_{e}^{\text {data }} \mathrm{T}_{e}^{\text {data }}+P_{e}^{\text {data }} \mathrm{T}_{e}^{\text {data }}+P_{e}^{\text {ack }} \mathrm{T}_{e}^{\text {ack }}+P_{e}^{\text {hack }} \mathrm{T}_{e}^{\text {hack }}
\end{gathered}
$$

For CR mode

$$
\begin{aligned}
& P_{e}^{r t s}=1-\left(1-P_{b}\right)^{\mathrm{L}_{\mathrm{rts}}} \\
& P_{e}^{c t s}=\left(1-P_{b}\right)^{\mathrm{L}_{\mathrm{trs}}}\left(1-\left(1-P_{b}\right)^{\mathrm{L}_{\mathrm{cts}}}\right) \\
& P_{e}^{\text {hcts }}=\left(1-P_{b}\right)^{\mathrm{L}_{\mathrm{rts}}+\mathrm{L}_{\mathrm{cts}}}\left(1-\left(1-P_{b}\right)^{\mathrm{L}_{\text {hcts }}}\right) \\
& P_{e}^{\text {data }}=\left(1-P_{b}\right)^{\mathrm{L}_{\mathrm{rts}}+\mathrm{L}_{\text {cts }}+\mathrm{L}_{\text {hcts }}}\left(1-\left(1-P_{b}\right)^{\mathrm{L}_{\text {data }}}\right) \\
& P_{e}^{\text {data' }}=\left(1-P_{b}\right)^{\mathrm{L}_{\mathrm{rts}}+\mathrm{L}_{\mathrm{cts}}+\mathrm{L}_{\text {hets }}+\mathrm{L}_{\text {data }}}\left(1-\left(1-P_{b}\right)^{\mathrm{L}_{\text {data }}}\right) \\
& P_{e}^{\text {ack }}=\left(1-P_{b}\right)^{\mathrm{L}_{\mathrm{rts}}+\mathrm{L}_{\mathrm{cts}}+\mathrm{L}_{\text {hets }}+\mathrm{L}_{\text {data }}+\mathrm{L}_{\text {data }}}\left(1-\left(1-P_{b}\right)^{\mathrm{L}_{\text {ack }}}\right)
\end{aligned}
$$



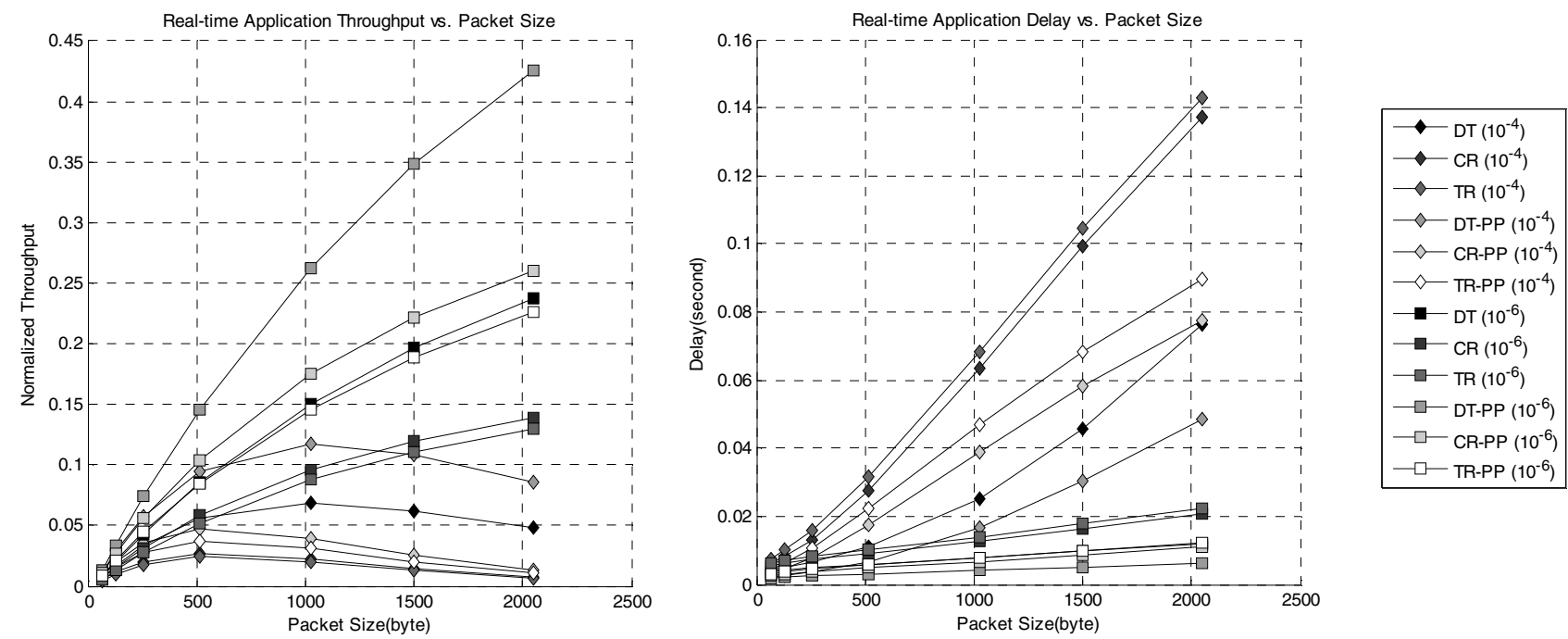

FIGURE 4: The real-time application throughput and delay performance comparison for under different packet sizes and channel bit error rates, when a realtime application and best-effort traffic compete with a channel.

$$
\begin{aligned}
& T_{e}^{r t s}=T_{r t s}+T_{c t s}^{\text {timeout }}+\delta+D I F S \\
& T_{e}^{c t s}=T_{r t s}+T_{c t s}+2 \delta+S I F S+D I F S \\
& T_{e}^{\text {hcts }}=T_{r t s}+T_{c t s}+\mathrm{T}_{\text {hcts }}+3 \delta+2 \text { SIFS }+ \text { DIFS } \\
& T_{e}^{\text {data }}=T_{r t s}+T_{\text {cts }}+T_{\text {hcts }}+T_{\text {data }}+T_{\text {data }}^{\text {timeout }}+ \\
& 4 \delta+3 S I F S+D I F S \\
& T_{e}^{\text {data' }}=T_{r \text { ts }}+T_{\text {cts }}+T_{\text {hcts }}+T_{\text {data }}+T_{\text {data' }} \\
& +T_{\text {ack }}^{\text {timeout }}+5 \delta+4 S I F S+D I F S \\
& T_{e}^{a c k}=T_{\text {rts }}+T_{\text {cts }}+T_{\text {hcts }}+T_{\text {data }}+T_{\text {data' }} \\
& +T_{a c k}+6 \delta+5 S I F S+D I F S \\
& T_{c}=T_{r t s}+\delta+D I F S \\
& T_{s}=T_{r t s}+T_{c t s}+T_{\text {hcts }}+T_{\text {data }}+T_{\text {data' }} \\
& +T_{\text {ack }}+6 \delta+5 \operatorname{SIFS}+\text { DIFS }
\end{aligned}
$$

where $\delta$ is the propagation delay, $\mathrm{T}_{\text {data }}$ is the data transmission time from the source node to the relay node, while $\mathrm{T}_{\text {data }^{\prime}}$ is the data transmission time from the relay node to the destination node. The ways to get $\mathrm{H}_{\mathrm{e}-\mathrm{DT}}$ and $\mathrm{H}_{\mathrm{e}-\mathrm{TR}}$ are nearly same.

$E\left[D_{i n t}\right]$, the average packet interval time between two successfully received packets at one receiver, can be obtained from the throughput expression by substituting equation (7):

$$
E\left[D_{\mathrm{int}}\right]=\frac{n \cdot E[p]}{S}=\frac{n \cdot E[\text { slot }]}{P_{t} P_{s}\left(1-P_{e}\right)}
$$

By combining this expression with equation- 7 and equation -8 , the above equation can be rewritten as:

$$
E\left[D_{\text {int }}\right]=\frac{E[\text { slot }]}{\tau(1-\tau)^{n-1}\left(1-P_{e}\right)}
$$

The average time to drop a packet can be expressed as:

$$
E\left[D_{\text {drop }}\right]=(m+1) T_{c}+E\left[X_{\text {drop }}\right] \cdot E[\text { slot }]
$$

where $E\left[X_{d r o p}\right]$ is the average number of slot times for a dropped packet after $m$ retransmissions.

Due to the fact that $P_{e}^{m+1}$ is the probability that a packet will finally be dropped after $m$ retransmissions, and the average number of time slots spent in the backoff stage $j$ is equal to $\frac{w_{j}+1}{2}, \mathrm{E}\left[\mathrm{X}_{\mathrm{drop}}\right]$ can be expressed as:

$$
E\left[X_{d r o p}\right]=\sum_{i=0}^{j} \frac{W_{i}+1}{2}
$$

For $\frac{P_{e}^{m+1}}{1-P_{e}^{m+1}}$ is average number of dropped packets relative to a successful transmission, the average packet delay in the saturated channel can be expressed as:

$$
E[D]=\sum_{j=0}^{m} T_{s}+j T_{c}+E[\text { slot }] E\left[\mathrm{X}_{\mathrm{drop}}\right] \frac{P_{e}^{(}\left(1-\mathrm{P}_{\mathrm{e}}\right)}{1-P_{e}^{m+1}}
$$

To Guarantee the QoS of real-time applications, in the preemptive mode, the guaranteed throughput of real-applications in a saturated channel can be expressed as:

$$
\mathrm{S}_{\text {realtitine }}=\frac{P_{t r} P_{s}\left(1-P_{e}\right) E[P]}{\left(1-P_{t r}\right) \sigma+P_{t r} P_{s}\left(1-P_{e}\right) T_{s}+P_{t r} P_{s} \mathrm{H}_{\text {precenpive }}+P_{t r} P_{s} \mathrm{H}_{\mathrm{e}}+P_{r}\left(1-P_{s}\right) T_{c}}
$$

where $H_{\text {preemptive }}$ is the operation overhead.

$$
\begin{aligned}
& H_{\text {preemptive-DT }}=H_{\text {preemptive-CR }}=T_{r t s}+\delta \\
& H_{\text {premptive-TR }}=T_{r t s}+P I F S+T_{h r t s}+\delta
\end{aligned}
$$

Assuming $R_{d}$ and $R_{c}$ are $11 \mathrm{Mbps}$ and $1 \mathrm{Mbps}$, respectively, the numerical results of the saturation throughput and delay performance of the real-time applications of ADC-MAC protocol with/without priority preemption (PP) can be calculated. Fig.4 shows both the theoretical throughput and delay performance comparison for three modes in the ADC- 
MAC protocol under different packet sizes and channel bit error rates when a real-time application and best-effort traffic compete with a saturated channel. It is observed that the priority preemption approach brings little more control overhead, however it can guarantee the bandwidth of real-time applications, and much reduce the transmission latency.

\section{CONCLUSION}

Our motivation for this research was to design a new adaptive distributed cooperative relay MAC protocol to improve the achieved system throughput, maximize the service range and guarantee the real-time application. In this paper, a novel cross-layer optimization approach based on our Distributed Cooperative Medium Access Control (ADC-MAC) protocol is developed and presented to guarantee realtime application QoS. Its performance is analyzed by Markov chain modeling, which shows that our proposed approach can effectively provide guaranteed QoS provisioning including bandwidth and delay for real-time applications by utilizing the concept of cooperative communications and exploiting spatial and user diversities.

\section{ACKNOWLEDGMENT}

This study was conducted by the Advanced Telecommunications Engineering Laboratory (TEL) (www.TEL.unl.edu) researchers at the University of Nebraska-Lincoln and it has been funded by the Federal Railroad Administration (FRA) under direction of Terry Tse. This study would not have been possible without the excellent support from Union Pacific Railroad, BNSF Railway, and Association of American Railroads (AAR).

\section{REFERENCES}

[1] Bryan Parno and Adrian Perrig, "Challenges in Securing Vehicular Networks", Proceedings of the Fourth Workshop on Hot Topics in Networks (HotNets-IV), November 14-15, 2005, College Park, MD.

[2] Hao Zhu, Guohong Cao, "rDCF: A Relay-Enabled Medium Access Control Protocol for Wireless Ad Hoc Networks," IEEE Transactions on Mobile Computing, vol. 5, no. 9, pp. 1201-1213, Sept. 2006.

[3] ANSI-IEEE 802.11 Standard : Wireless LAN Medium Access Control (MAC) and Physical Layer (PHY) Specifications 802.11, 1999.

[4] Pei Liu, Zhifeng Tao, Shivendra S. Panwar,"A cooperative mac protocol for wireless local area networks," in Proc. of IEEE ICC, (Seoul, Korea), June 2005.

[5] Pei Liu, Zhifeng Tao, Sathya Narayanan, Thanasis Korakis, ,"CoopMAC: A Cooperative MAC for Wireless LANs," IEEE Journal on Selected Areas in Communications, vol. 25, no. 2,pp. 340-354, February 2007.

[6] Haitao Zhao, Jibo Wei, Yong Xi, "A rate-adaptive cooperative MAC protocol based on RTS/CTS scheme for MANETs", ACCESSNETS 2007 pp.1-6 Aug. 2007.

[7] Jin Zhang, Qian Zhang and Weijia Jia, "A Novel MAC Protocol for Cooperative Downloading in Vehicular Networks", IEEE Globecom, 26-30 Nov. 2007 Page(s):4974 4978
[8] Jin Zhang, Qian Zhang and Weijia Jia, "VC-MAC: A cooperative MAC protocol in vehicular networks", IEEE Transcation on Vehicular Technology, vol.58, no.3, pp.15611571, 2009.

[9] T. Zhou, H. Sharif, M. Hempel, P. Mahasukhon, W. Wang, T. Ma, "A Novel Distributed Cooperative Relaying MAC Protocol for Vehicular Networks," accepted by IEEE JSAC, 2010.

[10] G. Bianchi, "Performance Analysis of the IEEE 802.11 Distributed Coordination Function." IEEE JSAC Vol.18, pp. 535-547, No.3, 2000.

[11] H. Wu, et, al. "Performance of Reliable Transport Protocol over IEEE 802.11 Wireless LAN: Analysis and Enhancement." INFOCOM. pp. 599-607, 2002.

[12] J. Yin, X. Wang, and D. P. Agrawal. "Optimal Packet Size in Error-prone Channel for IEEE 802.11 Distributed Coordination Function." WCNC. pp. 1654-1659, 2004.

[13] S. Ci, H. Sharif, and P. Mahasukhon, "Evaluating Saturation Throughput Performance of the IEEE 802.11 MAC under Fading Channels." IEEE BroadNets. . pp. 676-681, 2005.

[14] C. H. Foh, M. Zukerman, J. W. Tantra. "A Markovian Framework for Performance Evaluation of IEEE 802.11." IEEE Transactions on Wireless Communications. Vol. 6, pp. 1276-1265, 2007.

[15] http://www.atheros.com/pt/bulletins/AR5002X.pdf 